Original Research

\title{
Treatment satisfaction and its association with anxiety, depression and fear of COVID-19 among Lebanese inpatients with schizophrenia
}

\author{
Zeinab BITAR, Chadia HADDAD (D), Sahar OBEID iD, Souheil HALLIT (iD). \\ Received (first version): 18-Mar-2021 Accepted: 12-Sep-2021 Published online: 13-Sep-2021
}

\begin{abstract}
Background: The patient's evaluation of treatment and its associated outcomes define the treatment satisfaction. The quality of treatment satisfaction and healthcare service has been affected by depression, anxiety and fear of the current coronavirus disease 2019 (COVID-19) pandemic.

Objective: Therefore, this study aimed to assess factors associated with treatment satisfaction among Lebanese inpatients with schizophrenia, namely depression, anxiety and fear of COVID-19.

Methods: A cross-sectional study was conducted between September and November 2020, enrolled 118 patients with chronic schizophrenia consecutively admitted to Psychiatric Hospital of the Cross, Lebanon. The Functional Assessment of Chronic Illness Therapy-Treatment Satisfaction-Patient Satisfaction Scale (FACIT-TS-PS) was used to assess treatment satisfaction, the Lebanese Anxiety Scale -10 (LAS-10) was used to assess anxiety, Montgomery-Asberg Depression Rating Scale (MADRS) to assess depression and the Fear of COVID-19 Scale to assess the level of fear of the COVID-19 pandemic.

Results: The mean scores of the scales were as follows: treatment satisfaction (65.20; SD 16.11; median=71), LAS-10 (13.65; SD 6.02), MADRS (9.09; SD 6.69) and fear of COVID-19 (18.59; SD 6.78). Higher depression $(r=-0.46, p<0.001)$ was significantly associated with lower treatment satisfaction. Female gender (beta $=7.51, p=0.029$ ) was significantly associated with higher treatment satisfaction score. Fear of COVID-19 did not show any significant association with the treatment satisfaction score.

Conclusions: Results of this study found that depression and gender were associated with treatment satisfaction among inpatients with schizophrenia. No association has been found between fear of COVID-19 and treatment satisfaction among those patients. More research is warranted to evaluate treatment satisfaction and associated factors among chronic inpatients with schizophrenia,
\end{abstract} specifically during the COVID-19 pandemic, in order to improve treatment satisfaction and subjective well-being of patients.

\section{Keywords}

COVID-19; Schizophrenia; Patient Satisfaction; Fear; Anxiety Disorders; Anxiety; Depression; Inpatients; Risk Factors; Epidemiologic Factors; Multivariate Analysis; Cross-Sectional Studies; Lebanon

\section{INTRODUCTION}

Treatment is consisting of different elements such as individual psychotherapy, medication, and family intervention. ${ }^{1}$ The patient's evaluation of treatment and its associated outcomes define the treatment satisfaction. ${ }^{2}$ The clinical effectiveness of treatment comprise objective measures such as efficacy of drugs, safety, functioning and compliance with treatment, and subjective measures rated by the patients such as acceptability and satisfaction. ${ }^{3}$ Treatment satisfaction represents a reference point against which physician can evaluate their practice; it's an important indicator of the quality of healthcare in all settings, specifically in mental health. ${ }^{1,4}$ In general, patients with schizophrenia cannot determine how prevalent treatment side effects and symptoms are, so they associate their evaluation with their overall well-being. ${ }^{5}$ Also, a study

Zeinab BITAR. Faculty of Science, Lebanese University. Beirut (Lebanon).zaynab_bitar36@hotmail.com

Chadia HADDAD. Research Department, Psychiatric Hospital of the Cross. Jal Eddib (Lebanon). chadia_9@hotmail.com

Sahar OBEID*. Faculty of Arts and Science, Holy Spirit University of

Kaslik (USEK). Jounieh (Lebanon).saharobeid23@hotmail.com

Souheil HALLIT*${ }^{\star}$. Faculty of Medicine and Medical Sciences, Holy

Spirit University of Kaslik (USEK). Jounieh (Lebanon).

souheilhallit@hotmail.com

* Last co-authors

This work has been done in the frame of the "Behavioral and

Cognitive Neuroscience Master 2 program", Faculty of Sciences,

Lebanese University. has demonstrated that including patient with schizophrenia perspectives in the evaluation of their treatment can improve medical performance. ${ }^{6}$

During recent decades, the treatment of schizophrenia has evolved, the introduction of second-generation antipsychotics which generally have minimal side effects has been a principal pharmacological intervention. ${ }^{7}$ Studies revealed that patients with schizophrenia taking secondgeneration antipsychotics have higher treatment satisfaction compared with those taking first generation drugs, as well as receiving psychotherapy. ${ }^{8,9}$ Since poor adherence to treatment is associated with poor health outcomes such as a higher risk of relapse, rehospitalization, longer hospital admission, repeated emergency department visits, worsening of symptoms, and suicide attempts, the satisfaction with the medication in patients with schizophrenia is important for their adherence to antipsychotic treatment. ${ }^{8}$ Dua et al. showed that poor treatment satisfaction can lead to low treatment adherence among patients with schizophrenia. ${ }^{10}$

Communication represents the means by which the patient's symptoms are elicited, diagnosis is delivered, and treatment is recommended; it is the core of medical care and is important in all health care settings. ${ }^{11,12}$ Even though, it plays a crucial role in mental health care specifically in conditions that focus on hope, selfdetermination and self-efficacy, such as schizophrenia. ${ }^{13}$ 
The role of physician is to understand the experiences and the point of view of chronic patients with schizophrenia; and to include them in every decision about their treatment. ${ }^{13}$ A study showed a positive association between the providing of information about chronic illness and the maintenance of an effective therapy. ${ }^{14}$ Also, an important association between nurse communication and patients has been showed in recent studies. ${ }^{15}$ Regarding trust and confidence, patients who trust their physicians have shown increased treatment satisfaction and adherence. $^{16}$

In the scope of the schizophrenia disease, patients' satisfaction was associated negatively with depressive symptoms, which are in turn negatively associated with the subjective wellbeing. ${ }^{17}$ In addition, the content of delusions and hallucinations heard by patients with schizophrenia may affect their self-esteem, which has been recently associated with depression and anxiety and can affect patient's attitude to medication and treatments. ${ }^{18,19}$

The coronavirus disease (COVID-19) pandemic continues to grow, and as of January 24, 2021, there have been $97,464,094$ confirmed cases of COVID-19, including $2,112,689$ deaths, reported to World Health Organization (WHO). ${ }^{20}$ Deaths of family members, friends, or colleagues; financial insecurity; unemployment and isolation from others are significant psychological stressors causing depression, worsening of public mental health and intensify fear of the virus. ${ }^{21}$ Researchers showed that the stress caused by fear of COVID-19 might be higher and worse among patients with schizophrenia. ${ }^{22}$ The pandemic has created challenges for the health care professionals, specifically for those providing services to patients with schizophrenia. ${ }^{22}$ These challenges has consequently affected the satisfaction of chronic patients. ${ }^{23}$

Lebanon is lurching from crisis to crisis, making long term plans to develop mental health sector very difficult to be reached, poorly prepared to meet the challenges such as the current COVID-19 pandemic and putting pressure on existing treatments. Less attention has been given to the effect of the COVID-19 pandemic on treatment satisfaction, specifically among patients with schizophrenia. The main objective of this study, the first of its kind in Lebanon to the best of our knowledge, was to assess treatment satisfaction among patients with schizophrenia and its association with anxiety, depression and fear of COVID-19.

\section{METHODS}

\section{Study design and sample size}

This cross-sectional study was carried out between September and November 2020, enrolled all chronic patients with schizophrenia admitted to the hospital, hospitalized for more than one year, aged above 18 years, and all treated in the Psychiatric Hospital of the Cross, the largest psychiatric hospital in Lebanon. After eligibility criteria were determined, our study included 118 participants with a clinically confirmed diagnosis of schizophrenia (based on the Diagnostic and Statistical Manual $5^{\text {th }}$ Edition DSM-5 criteria). Patients were excluded if their clinical diagnosis performed by physicians prevented them from participating in the interviews; exclusions are consisting of patients with schizoaffective (based on the Diagnostic and Statistical Manual $5^{\text {th }}$ Edition DSM-5 criteria), cognitive impairment (based on the Mini-Mental State Exam MMSE), delusional (patients unable to answer correctly) and those who refused to participate.

The Epi info software (Centers for Disease Control and Prevention, Epi Info) was used to calculate the required sample size. Considering a psychiatric patients of 40,000 and a prevalence of $69 \%$ of patients were satisfied with their treatment, with a $90 \%$ confidence level and an alpha error of $7 \%$ the minimal sample size needed was $118 .{ }^{10}$

\section{Data collection and measurement}

The preparation of the questionnaire was based on the fact that Arabic is the native language in Lebanon, with an average time between 30 and 45 min needed to complete it. One trained research assistant was responsible for the data collection, via a personal interview with each patient. Questions covered different aspects; the first part included socio-demographic characteristics (gender, age, region, marital status, educational level). The second part included questions about medical history and medication.

The treatment satisfaction is rated by Functional Assessment of Chronic Illness Therapy-Treatment Satisfaction-Patient Satisfaction (FACIT-TS-PS), which is divided to 5 subscales: physician communication (12 items), treatment staff communication (4 items), technical competence ( 3 items), confidence and trust ( 4 items), nurse communication ( 3 items), and general questions ( 3 items). Each of the five subscales are coded on a 4-point scale: $0=$ no, not at all, 1=yes, but not as much as I wanted, 2=yes, almost as much as I wanted and $3=y e s$, and as much as I wanted; and for the general questions it's a 3-point scale: 0=no, 1=maybe, 2=yes. Higher FACIT-TS-PS scores represent higher treatment satisfaction. ${ }^{24}$ The Arabic version of FACIT-TS-PS used in our study was translated by FACIT translator. ${ }^{25}$ In this study, the Cronbach's alpha for the total scale was 0.932 , for each subscales was: physician communication (Cronbach's alpha $=0.966$ ), treatment staff communication (Cronbach's alpha=0.499), technical competence (Cronbach's alpha $=0.875$ ), confidence and trust (Cronbach's alpha $=0.947$ ), nurse communication

\begin{tabular}{|c|c|}
\hline \multicolumn{2}{|l|}{ Gender; n (\%) } \\
\hline Female & $60(50.8)$ \\
\hline Male & $58(49.2)$ \\
\hline \multicolumn{2}{|l|}{ Education level; n (\%) } \\
\hline Illiterate & $16(14.0)$ \\
\hline Elementary & 17 (14.9) \\
\hline Intermediate & $31(27.2)$ \\
\hline Secondary & $29(25.4)$ \\
\hline University & $21(18.4)$ \\
\hline \multicolumn{2}{|l|}{ Marital status; n (\%) } \\
\hline Single & $108(91.5)$ \\
\hline Married & $10(8.5)$ \\
\hline \multicolumn{2}{|l|}{ Region; n (\%) } \\
\hline Beirut & $51(45.9)$ \\
\hline Mount Lebanon & $15(13.5)$ \\
\hline North Lebanon & $26(23.4)$ \\
\hline South Lebanon & $7(6.3)$ \\
\hline Beqaa & $12(10.8)$ \\
\hline Age in years; mean (SD) & 51.78 (14.61) \\
\hline
\end{tabular}




\begin{tabular}{|c|c|c|c|c|}
\hline \multicolumn{2}{|l|}{ Table 2. Description of the scales used in the study } & Median & Minimum & Maximum \\
\hline & Mean (SD) & 71.00 & 0 & 106.00 \\
\hline Treatment satisfaction total score & $65.20(16.11)$ & 35.00 & 0 & 36.00 \\
Physician communication & $29.76(8.91)$ & 12.00 & 0 & 9.00 \\
Treatment staff communication & $10.25(4.13)$ & 9.00 & 0 & 12.00 \\
Technical competence & $7.55(2.15)$ & 12.00 & 0 & 9.00 \\
Confidence and trust & $10.04(2.84)$ & 9.00 & 5 & 35.00 \\
Nurse communication & $7.59(2.03)$ & 12.00 & 0 & 38.00 \\
\hline Lebanese Anxiety Scale -10 (LAS-10) & $13.65(6.02)$ & 8.00 & 0 & 35.00 \\
\hline Montgomery-Asberg Depression Rating & $9.09(6.69)$ & & 15.00 & 0 \\
Scale (MADRS) & & & 0 \\
\hline Fear of COVID-19 scale & $18.59(6.78)$ & & & 0 \\
\hline
\end{tabular}

(Cronbach's alpha=0.854) and general questions (Cronbach's alpha=0.303).

The Lebanese Anxiety Scale -10 (LAS-10) [26] consists of 10 items to measure the severity of anxiety symptoms on a sample of Lebanese individuals. Higher scores reflect more anxiety. In this study, the Cronbach's alpha was 0.814 .

Depression is rated by Montgomery-Asberg Depression Rating Scale (MADRS), it consists of 10 items, and each defined by a series of symptoms, to measure the severity of depression. ${ }^{27}$ The Arabic MADRS version is a validated and reliable tool to estimate the overall severity of depression among psychiatric patients in Lebanon. ${ }^{28}$ Higher scores represent more depressive symptoms. ${ }^{28}$ In this study, the Cronbach's alpha was 0.781 .

The Fear of COVID-19 Scale (FCV-19S) assess one's fear levels of COVID-19. It consists of 7 items and is scored on a 5 -point scale, ranging from 1 (strongly disagree) to 5 (strongly agree). A total score is calculated by summing all item scores with a possible total score ranging between 7 and 35. Higher scores indicate greater levels of fear of COVID-19. ${ }^{29}$ Validated Arabic version of FCV-19S was used in our study. In this study, the Cronbach's alpha was 0.911 .

\section{Statistical analysis}

The FACTOR software was used to conduct the factor analysis, using the Pearson matrix correlation and using the parallel analysis as a procedure for determining the number of factors/components. The promax rotation was used to extract the items since the latter were highly correlated. The Kaiser-Meyer-Olkin (KMO) and the Bartlett's test of sphericity $p$-value were calculated to ensure model's adequacy.

The SPSS software version 25.0 was used for all statistical analysis. A Cronbach's alpha was recorded for reliability analysis for all the scales. In descriptive analyses, means and standard deviations were presented for continuous variables, while percentages were presented for categorical variables. The mean percentage of missing data was less than $5.0 \%$ of the database therefore, we did not replace those values. To compare continuous variables between two groups, the Student t-test was used, the ANOVA F tests was used to compare continuous variables between more than two groups. Pearson correlation was used for linear correlation between continuous variables. A stepwise linear regressions was conducted, taking the treatment satisfaction variable as the dependent variables. Variables that showed a $p<0.25$ in the bivariate analysis were entered in the final model. ${ }^{30} \mathrm{~A} p<0.05$ was considered significant in all cases.

\section{RESULTS}

A total of 118 (55.14\%) patients out of 214 eligible to participate was enrolled in this study. The sociodemographic characteristics of the participants were summarized in Table 1 . The results showed that the mean age of the participants was 51.78, SD 14.61 years. Half of the sample was female (50.8\%), the majority was single (91.5\%). Only $18.4 \%$ have a university education level and $45.9 \%$ were from Beirut region.

The scales description was summarized in Table 2 . The mean scores of the scales were as follows: treatment satisfaction (65.20; SD 16.11; median=71), LAS-10 (13.65; SD 6.02), MADRS (9.09; SD 6.69) and fear of COVID-19 (18.59; SD 6.78).

A factor analysis, using the principal component analysis, was conducted on the treatment satisfaction scale items (Table 3). The overall items were removed because of low communality $(<0.3)$. The other remaining items converged into two factors (Factor 1 "physician and treatment staff communication, confidence and trust, and technical competence"; Factor 2 "nurse communication"). The KMO value $=0.943$ (very good) and the Bartlett test of sphericity $p<0.001$ ensured the model adequacy. The items were able to explain $88.5 \%$ of the variance (Table 2). Moreover, the inter-factor correlation was adequate $(r=0.495)$.

The unadjusted results showed that higher treatment satisfaction scores were found in males compared to females (beta $=12.35, p<0.001$ ). Higher anxiety (beta $=-1.20$, $p<0.001)$ and higher depression (beta $=-1.12, p<0.001$ ) were significantly associated with lower treatment satisfaction (Table 4).

When adjusting the results over all variables that showed a $p<0.25$, the results of the forward linear regression showed that higher depression (beta $=-0.68, p=0.007$ ) was significantly associated with lower treatment satisfaction, whereas males (beta $=8.59, p=0.005$ ) had more treatment satisfaction compared to females (Table 4).

\section{DISCUSSION}

During the current COVID-19 pandemic, the quality of treatment satisfaction and healthcare service has been affected by many factors. Therefore, this study aimed to assess factors associated with treatment satisfaction among Lebanese inpatients with schizophrenia, namely depression, anxiety and fear of COVID-19. Our results showed that treatment satisfaction was correlated with depression and gender. 


\begin{tabular}{|c|c|c|c|c|}
\hline Variable & Factor 1 & Factor 2 & Eigenvalue & $\begin{array}{c}\text { h2 } \\
\text { communality }\end{array}$ \\
\hline PC1: Did your doctor(s) give explanations that you could understand? & 1.012 & & 2.17 & 0.962 \\
\hline PC2: Did your doctor(s) explain the possible benefits of your treatment? & 1.006 & & 1.94 & 0.959 \\
\hline $\begin{array}{l}\text { PC3: Did your doctor(s) explain the possible side effects or risks of your } \\
\text { treatment? }\end{array}$ & 0.980 & & 1.80 & 0.919 \\
\hline PC4: Did you have an opportunity to ask questions? & 1.011 & & 1.69 & 0.967 \\
\hline PC5: Did you get to say the things that were important to you? & 1.010 & & 1.60 & 0.962 \\
\hline PC6: Did your doctor(s) seem to understand what was important to you? & 1.006 & & 1.50 & 0.967 \\
\hline PC7: Did your doctor(s) show genuine concern for you? & 1.005 & & 1.41 & 0.973 \\
\hline PC8: Did your doctor(s) seem to understand your needs? & 0.994 & & 1.34 & 0.945 \\
\hline PC9: Were you able to talk to your doctor(s) when you needed to? & 1.015 & & 1.27 & 0.977 \\
\hline PC10: Were you encouraged to participate in decisions about your health care? & 0.989 & & 1.20 & 0.894 \\
\hline PC11: Did you have enough time to make decisions about your health care? & 1.003 & & 1.13 & 0.945 \\
\hline PC12: Did your doctor(s) seem to respect your opinions? & 1.012 & & 1.07 & 0.958 \\
\hline $\begin{array}{l}\text { TSC1: Did the treatment staff discuss how your health and treatment may affect } \\
\text { your normal work (including housework)? }\end{array}$ & 0.905 & & 1.01 & 0.857 \\
\hline $\begin{array}{l}\text { TSC2: Did the treatment staff discuss how your health and treatment may affect } \\
\text { your normal daily activities? }\end{array}$ & 0.943 & & 0.96 & 0.910 \\
\hline $\begin{array}{l}\text { TSC3: Did the treatment staff discuss how your health and treatment may affect } \\
\text { your personal relationships? }\end{array}$ & 0.936 & & 0.89 & 0.861 \\
\hline $\begin{array}{l}\text { TSC4: Did the treatment staff discuss how your health and treatment may affect } \\
\text { you emotionally? }\end{array}$ & 0.941 & & 0.85 & 0.915 \\
\hline TCC1: Did you feel your doctor(s) had experience treating your illness? & 0.915 & & 0.80 & 0.874 \\
\hline $\begin{array}{l}\text { TCC2: Did you feel your doctor(s) knew about the latest medical developments for } \\
\text { your illness? }\end{array}$ & 0.846 & & 0.74 & 0.788 \\
\hline TCC3: Was the treatment staff thorough in examining and treating you? & 0.825 & & 0.69 & 0.711 \\
\hline NC1: 1 Did your nurse(s) give explanations that you could understand? & & 0.957 & 0.65 & 0.865 \\
\hline NC2: Did your nurse(s) show genuine concern for you? & & 0.910 & 0.60 & 0.829 \\
\hline NC3: Did your nurse(s) seem to understand your needs? & & 0.947 & 0.55 & 0.818 \\
\hline CANDT1: Did you feel that the treatment staff answered your questions honestly? & 0.697 & & 0.51 & 0.803 \\
\hline CANDT2: Did the treatment staff respect your privacy? & 0.740 & & 0.46 & 0.823 \\
\hline CANDT3: Did you have confidence in your doctor(s)? & 0.671 & & 0.42 & 0.735 \\
\hline CANDT4: Did you trust your doctor(s)' suggestions for treatment? & 0.728 & & 0.36 & 0.801 \\
\hline Percentage of variance explained & 76.5 & 12.0 & & \\
\hline Cronbach's alpha & 99.7 & 95.0 & & \\
\hline
\end{tabular}

In accordance with previous studies, our results showed higher depression was associated with lower treatment satisfaction among patients with schizophrenia. ${ }^{31}$ These results can be justified by many explanations. First, patients with schizophrenia present poor insight, which has been associated with increased depression and less positive attitudes to medication and treatments. ${ }^{19,32}$ Secondly, the content of delusions and disparaging voices heard by patients with schizophrenia may affect their self-esteem, which has been recently associated with depression. ${ }^{18}$

Our results showed no association between fear of COVID19 and treatment satisfaction among patients with schizophrenia. To our knowledge, no other study conducted so far has evaluated patients' satisfaction in similar framework, so we couldn't compare our findings to other studies. Our results can be justified by many factors. First, our study was conducted on September 2020 where the total number of recorded cases -since February 2020 when the first covid-19 case was recorded in Lebanon- was only 469 cases. $^{33}$ Secondly, during the pandemic, the hospital used high safety measures and precautions to protect the inpatients from the virus. Third, a safety protocol was used to limit the access of people from the exterior. These factors can lead to decrease the fear of COVID-19 among inpatients in our sample. However, further studies will be needed to evaluate this association specifically after the fast increasing rate of cases during the past few weeks.
Also, our findings showed a significant association between gender and treatment satisfaction. The effect of gender on treatment satisfaction among psychiatric patients differs among previous studies; some studies has shown no clear association with patient satisfaction. ${ }^{34}$ In contrast, some studies showed that female patients with schizophrenia are dissatisfied with their treatment; this dissatisfaction can be due to cultural limitations in Arab world where women are more conservative and their exposure to the public is limited. ${ }^{35}$ Also, kuosmanen et al. revealed an association between gender, duration of mental illnesses and satisfaction with the staff-patient relationship; with no clear justification of this correlation. More research will be needed to clarify our findings and study why females were more satisfied than men, in order to ensure more individual medical care. ${ }^{36}$

There is a need to further evaluate and understand the treatment priorities, goals and satisfaction from the perspective of patients with schizophrenia; this could improve service adherence, have better treatment outcomes and subjective well-being, specifically during the COVID-19 pandemic.

\section{Limitations and strengths}

Many limitations could be raised in our study. First, this is a cross-sectional study and cause-effect relationships are not allowed. Second, the COVID-19 pandemic and the 


\begin{tabular}{|c|c|c|c|c|c|c|c|c|}
\hline & \multicolumn{4}{|c|}{ Unadjusted results } & \multicolumn{4}{|c|}{ Adjusted results** } \\
\hline & beta & $\beta$ & p & $95 \% \mathrm{Cl}$ & beta & $\beta$ & p & $95 \% \mathrm{Cl}$ \\
\hline Gender (males vs females*) & 12.35 & 0.39 & $<0.001$ & $6.90-17.80$ & 8.59 & 0.27 & 0.005 & $2.67-14.51$ \\
\hline Depression & -1.12 & -0.47 & $<0.001$ & $-1.51--0.73$ & -0.68 & -0.26 & 0.007 & $-1.17--0.19$ \\
\hline Anxiety & -1.20 & -0.45 & $<0.001$ & $-1.64--0.76$ & & & & \\
\hline Fear of COVID-19 & -0.35 & -0.15 & 0.124 & $-0.79-0.10$ & & & & \\
\hline Age & 0.004 & 0.004 & 0.967 & $-0.20-0.21$ & & & & \\
\hline Marital status (married vs single*) & -6.12 & -0.11 & 0.252 & $-16.66-4.42$ & & & & \\
\hline YYEducation level & & & & & & & & \\
\hline Illiterate* & - & - & - & - & & & & \\
\hline Elementary & 4.03 & 0.09 & 0.321 & $-3.98-12.04$ & & & & \\
\hline Intermediate & -5.04 & -0.15 & 0.120 & $-11.41-1.33$ & & & & \\
\hline Secondary & 1.14 & 0.03 & 0.732 & $-5.44-7.72$ & & & & \\
\hline University & 0.20 & 0.01 & 0.958 & $-7.20-7.59$ & & & & \\
\hline
\end{tabular}

lockdown limited us from a total data collection; the sample size was relatively small despite having enough statistical power for the bivariate and multivariable analysis. In addition, patients were recruited from one hospital in Lebanon, predisposing us to a selection bias. Some questions might have been over- or underestimated by the patients, leading to a possible information bias. A residual confounding bias is possible since not all factors associated with treatment satisfaction were included in the questionnaire. However, our study was the first to evaluate the treatment satisfaction and its association with psychopathology during the COVID-19 pandemic and among chronic inpatients with schizophrenia using different validated scales (LAS-10 and MADRS).

\section{CONCLUSIONS}

Results of this study found that lower treatment satisfaction was associated with higher depression among patients with schizophrenia. Gender plays a major role in the evaluation of treatment satisfaction. No association has been found between fear of COVID-19 and treatment satisfaction among inpatients with schizophrenia. More research is warranted to evaluate treatment satisfaction and associated factors among patients with schizophrenia, specifically during the COVID-19 pandemic, in order to improve treatment satisfaction and subjective well-being of patients. Finally, during this hard and unstable situation in Lebanon, the challenge remains for physicians to take all patients' needs into consideration by adopting new communication methods and strategies besides clinical diagnosis to ensure a good medical care procedure and effective treatment outcomes.

\section{ACKNOWLEDGEMENTS}

We would like to thank all patients who agreed to participate in this study.

\section{CONFLICT OF INTEREST}

The authors have nothing to disclose.

\section{FUNDING}

None.

\section{AUTHOR ROLES (CRediT)}

Conceptualization: $\mathrm{SH}, \mathrm{SO}$.

Data curation: ZB.

Formal analysis: $\mathrm{CH}, \mathrm{SH}$.

Investigation: $\mathrm{ZB}, \mathrm{CH}, \mathrm{SO}, \mathrm{SH}$.

Methodology: $\mathrm{ZB}, \mathrm{CH}, \mathrm{SO}, \mathrm{SH}$.

Supervision: $\mathrm{SH}$

Validation: $\mathrm{SH}, \mathrm{SO}$.

Writing - original draft: ZB.

Writing - review \& editing: SO, SH.

\section{References}

1. Haahr U, Simonsen E, Røssberg Jl, et al. Patient satisfaction with treatment in first-episode psychosis. Nord J Psychiatry 2012;66(5):329-335. https://doi.org/10.3109/08039488.2011.644808

2. Shikiar R, Rentz AM. Satisfaction with medication: an overview of conceptual, methodologic, and regulatory issues. Value Health. 2004;7(2):204-215. https://doi.org/10.1111/j.1524-4733.2004.72252.x

3. Peuskens J. Clinical effectiveness in adults with chronic schizophrenia. Eur Neuropsychopharmacol. 2004;14 Suppl 4:S453-S459. https://doi.org/10.1016/..euroneuro.2004.08.004

4. Chue $P$. The relationship between patient satisfaction and treatment outcomes in schizophrenia. J Psychopharmacol. 2006;20(6 Suppl):38-56. https://doi.org/10.1177/1359786806071246

5. Carrick R, Mitchell A, Powell RA, Lloyd K. The quest for well-being: a qualitative study of the experience of taking antipsychotic medication. Psychol Psychother. 2004;77(Pt 1):19-33. https://doi.org/10.1348/147608304322874236

6. Afe T, Bello-Mojeed M. Schizophrenia treatment satisfaction in Nigeria. Psychiatr Serv. 2014;65(5):697-698. https://doi.org/10.1176/appi.ps.650502

7. Ladea M, Barbu CM, Juckel G. Treatment effectiveness in patients with schizophrenia as measured by the ASSESS battery - first longitudinal data. Psychiatr Danub. 2015;27(4):364-370. 
8. Fujikawa M, Togo T, Yoshimi A, et al. Evaluation of subjective treatment satisfaction with antipsychotics in schizophrenia patients. Prog Neuropsychopharmacol Biol Psychiatry. 2008;32(3):755-760. https://doi.org/10.1016/i.pnpbp.2007.12.002

9. Stabell LA, Gjestad R, Kroken RA, Løberg EM, Jørgensen HA, Johnsen E. Predictors of treatment satisfaction in antipsychotic-naïve and previously medicated patients with acute-phase psychosis. Nord J Psychiatry. 2019;73(6):349356. https://doi.org/10.1080/08039488.2019.1636134

10. Dua D, Hertz D, Howe T. Overall treatment satisfaction with schizophrenia therapies: analysis from patient, physician and caregiver perspectives. Value Health. 2018;21:S186--S187. https://doi.org/10.1016/j.jval.2018.04.1294

11. McCabe R, Healey PGT. Miscommunication in Doctor-Patient Communication. Top Cogn Sci. 2018;10(2):409-424. https://doi.org/10.1111/tops.12337

12. Pestana-Santos A, Loureiro L, Santos V, Carvalho I. Patients with schizophrenia assessing psychiatrists' communication skills. Psychiatry Res. 2018;269:13-20. https://doi.org/10.1016/i.psychres.2018.08.040

13. Schneider B, Scissons H, Arney L, et al. Communication between people with schizophrenia and their medical professionals: a participatory research project. Qual Health Res. 2004;14(4):562-577. https://doi.org/10.1177/1049732303262423

14. Hornung WP, Klingberg S, Feldmann R, Schonauer K, Schulze Mönking H. Collaboration with drug treatment by schizophrenic patients with and without psychoeducational training: results of a 1-year follow-up. Acta Psychiatr Scand. 1998;97(3):213-219. https://doi.org/10.1111/j.1600-0447.1998.tb09990.x

15. Lotfi M, Zamanzadeh V, Valizadeh L, Khajehgoodari M. Assessment of nurse-patient communication and patient satisfaction from nursing care. Nurs Open. 2019;6(3):1189-1196. https://doi.org/10.1002/nop2.316

16. Alimena S, Air ME. Trust, Satisfaction, and Confidence in Health Care Providers Among Student and Professiona Dancers in France. Med Probl Perform Art. 2016;31(3):166-173. https://doi.org/10.21091/mppa.2016.3030

17. Kim JH, Ann JH, Kim MJ. Relationship between improvements of subjective well-being and depressive symptoms during acute treatment of schizophrenia with atypical antipsychotics. J Clin Pharm Ther. 2011;36(2):172-178. https://doi.org/10.1111/j.1365-2710.2010.01175.x

18. McCabe R, Saidi M, Priebe S. Patient-reported outcomes in schizophrenia. Br J Psychiatry. 2007;50(suppl):s21-s28. https://doi.org/10.1192/bjp.191.50.s21

19. Freudenreich O, Cather C, Evins AE, Henderson DC, Goff DC. Attitudes of schizophrenia outpatients toward psychiatric medications: relationship to clinical variables and insight. J Clin Psychiatry. 2004;65(10):1372-1376. https://doi.org/10.4088/icp.v65n1012

20. WHO Coronavirus Disease (COVID-19) Dashboard. https://covid19.who.int (accessed Jan 24, 2021)

21. Shader RI. COVID-19 and Depression. Clin Ther. 2020;42(6):962-963. https://doi.org/10.1016/i.clinthera.2020.04.010

22. Kozloff N, Mulsant BH, Stergiopoulos V, Voineskos AN. The COVID-19 Global Pandemic: Implications for People With Schizophrenia and Related Disorders. Schizophr Bull. 2020;46(4):752-757. https://doi.org/10.1093/schbul/sbaa051

23. Deriba BS, Geleta TA, Beyane RS, Mohammed A, Tesema M, Jemal K. Patient Satisfaction and Associated Factors During COVID-19 Pandemic in North Shoa Health Care Facilities. Patient Prefer Adherence. 2020;14:1923-1934. https://doi.org/10.2147/ppa.s276254

24. Peipert JD, Beaumont JL, Bode R, Cella D, Garcia SF, Hahn EA. Development and validation of the functional assessment of chronic illness therapy treatment satisfaction (FACIT TS) measures. Qual Life Res. 2014;23(3):815-824 https://doi.org/10.1007/s11136-013-0520-8

25. FACIT Group. FACIT-TS-PS Languages. https://www.facit.org/measure-languages/FACIT-TS-PS-Languages (accessed Feb 8, 2021).

26. Hallit S, Obeid S, Haddad C, et al. Construction of the Lebanese Anxiety Scale (LAS-10): a new scale to assess anxiety in adult patients. Int J Psychiatry Clin Pract. 2020;24(3):270-277. https://doi.org/10.1080/13651501.2020.1744662

27. Montgomery SA, Asberg M. A new depression scale designed to be sensitive to change. Br J Psychiatry. 1979;134:382389. https://doi.org/10.1192/bjp.134.4.382

28. Hallit S, Obeid S, El Hage W, Kazour F. Validation of the Arabic version of the MADRS scale among Lebanese patients with depression. Encephale. 2019;45(3):195-199. https://doi.org/10.1016/i.encep.2018.05.004

29. Alyami M, Henning M, Krägeloh CU, Alyami H. Psychometric Evaluation of the Arabic Version of the Fear of COVID-19 Scale. Int J Ment Health Addict. 2020; [ahead of print] https://doi.org/10.1007/s11469-020-00316-X

30. Bursac Z, Gauss CH, Williams DK, Hosmer DW. Purposeful selection of variables in logistic regression. Source Code Biol Med. 2008;3:17. https://doi.org/10.1186/1751-0473-3-17

31. Fakhoury WK, Wright D, Wallace M. Prevalence and extent of distress of adverse effects of antipsychotics among callers to a United Kingdom National Mental Health Helpline. Int Clin Psychopharmacol. 2001;16(3):153-162. https://doi.org/10.1097/00004850-200105000-00004

32. Karow A, Pajonk FG. Insight and quality of life in schizophrenia: recent findings and treatment implications. Curr Opin Psychiatry. 2006;19(6):637-641. https://doi.org/10.1097/01.yco.0000245754.21621.c9

33. Worldometer. Lebanon Coronavirus: 282,249 cases and 2,374 deaths. https://www.worldometers.info/coronavirus/country/lebanon/ (accessed Jan 25, 2021).

34. Middelboe T, Schjødt T, Byrsting K, Gjerris A. Ward atmosphere in acute psychiatric in-patient care: patients' perceptions, ideals and satisfaction. Acta Psychiatr Scand. 2001;103(3):212-219. https://doi.org/10.1034/j.1600-0447.2001.00102.x

35. Bener A, Ghuloum S. Gender difference on patients' satisfaction and expectation towards mental health care. Niger J Clin Pract. 2013;16(3):285-291. https://doi.org/10.4103/1119-3077.113448

36. Kuosmanen L, Hätönen $\mathrm{H}$, Jyrkinen AR, Katajisto J, Välimäki M. Patient satisfaction with psychiatric inpatient care. J Adv Nurs. 2006;55(6):655-663. https://doi.org/10.1111/i.1365-2648.2006.03957.x 